\section{Selection Diversity Receivers Over Nonidentical Weibull Fading Channels}

\author{
Nikos C. Sagias, Dimitris A. Zogas, and George K. Karagiannidis
}

\begin{abstract}
The performance of selection combining (SC) receivers operating over independent, but not necessarily identically distributed, Weibull fading channels is studied. A novel closed form expression for the moments of the SC output signal-to-noise ratio (SNR) is derived, which is used to study the corresponding average output SNR and amount of fading. Second-order statistical parameters such as the average level crossing rate and average fade duration at the output of the $\mathrm{SC}$ are also obtained in closed form. Moreover, the average symbol error probability for several coherent and noncoherent modulations schemes as well as the Shannon capacity are extracted in terms of the tabulated Meijer's G-function. Simulations are also performed to validate the proposed formulation.
\end{abstract}

Index Terms-Average fade duration (AFD), average level crossing rate (LCR), average symbol error probability (ASEP), selection combining (SC), Shannon capacity, Weibull fading channels.

\section{INTRODUCTION}

Various well-known diversity techniques, such as selection combining (SC), equal-gain combining (EGC), maximal-ratio combining (MRC), and generalized-selection combing (GSC) are used in wireless digital communications systems to mitigate the detrimental effects of channel fading. Among these techniques, SC requires the lowest implementation complexity at the expense of performance providing. In selection diversity, the branch with the highest instantaneous signal-to-noise ratio (SNR) is selected among $L$ available [1]. The performance of SC receivers has been extensively studied in the open technical literature for several well-known fading statistical models, such as Rayleigh and Nakagami- $m$, for both independent and correlative fading [1]-[7]. Published papers concerning the performance of digital receivers over Weibull fading channels, with or without, diversity are scarce. The most important contributions among them are [8]-[12]. More specifically, the performance of SC receivers operating over independent Weibull fading has been investigated in [10] and [12]. However, to the best of the authors' knowledge, the performance of SC receivers has not been addressed yet considering a more realistic and practical fading scenario with independent, but not necessarily identically distributed, input branches.

The structure and the contribution of this paper are as follows. In Section II, a useful and mathematical tractable expression for the probability density function (PDF) of the SC output SNR is extracted. Using this PDF formula, novel closed-form expressions for the moments of the output SNR are derived and used to study important performance criteria, such as the average SNR and amount of fading (AoF) at the output of the SC. In Section III, closed-form expressions for the average level crossing rate (LCR) and average fade duration (AFD) at the output of the combiner are obtained. The average symbol error

Manuscript received August 5, 2004; revised October 19, 2004 and January 14,2005 . This paper was presented in part at the IEEE Global Telecommunications Conference, Dallas, TX, December 2004. The review of this paper was coordinated by Prof. Y. Ma.

N. C. Sagias is with the Laboratory of Electronics, Department of Physics, University of Athens, GR-15784 Athens, Greece (e-mail: nsagias@space. noa.gr).

D. A. Zogas is with the Electrical and Computer Engineering Department, University of Patras, Rion, GR-26442 Patras, Greece (e-mail: zogas@ space.noa.gr).

G. K. Karagiannidis is with the Division of Telecommunications, Electrical and Computer Engineering Department, Aristotle University of Thessaloniki, GR-54124 Thessaloniki, Greece (e-mail: geokarag@auth.gr).

Digital Object Identifier 10.1109/TVT.2005.853452 probability (ASEP) for several modulations schemes and the average channel capacity are expressed in terms of the tabulated Meijer's G-function [13, eq. (9.301)] in Sections IV and V, respectively. Sections II-V contain several selected numerical examples, which outline the mathematical analysis and show the effects of various channel and system parameters, such as the fading severity, the power delay profile (PDP), and the number of diversity branches on the combiner performance. Finally, the main points are summarized in Section VI.

\section{STATISTICS OF THE SC OUTPUT SNR}

We consider an $L$-branch SC receiver operating over a Weibull fading environment. In each branch, fading is described by the Weibull distribution which considers a signal composed of clusters of one multipath wave each propagating in a nonhomogeneous environment [14]. Suppose that such a nonlinearity is in the form of a power, so that the resulting envelope is observed as the modulus of the multipath Rayleigh component $\left\{X_{\ell}\right\}_{\ell=1}^{L}$ to the power of $2 / \beta_{l}$. Hence, the received envelope in the $\ell$ th diversity branch is

$$
R_{\ell}=X_{\ell}^{2 / \beta_{\ell}} .
$$

In the above equation, $R_{\ell}$ is a Weibull distributed random variable (RV) with cumulative distribution function (CDF)

$$
F_{R_{\ell}}(r)=1-\exp \left(-\frac{r^{\beta_{\ell}}}{\Omega_{\ell}}\right)
$$

where $\Omega_{\ell}$ is related to the average fading power $\mathcal{E}\left\langle R_{\ell}^{2}\right\rangle$ as $\Omega_{\ell}^{2 / \beta_{\ell}}=$ $\mathcal{E}\left\langle R_{\ell}^{2}\right\rangle / \Gamma\left(1+2 / \beta_{\ell}\right)$, with $\Gamma(\cdot)$ being the Gamma function [13, eq. (8.310/1)]. Moreover, $\beta_{\ell}>0$ is the fading parameter expressing the severity of fading. For the special case of $\beta_{\ell}=2$, the Rayleigh model may be considered.

The instantaneous envelope $R$ at the output of the SC receiver will be the one with the highest instantaneous value among the $L$ branches, i.e.,

$$
R=\max \left\{R_{\ell}\right\} .
$$

The CDF of $R$ equals to the probability $\operatorname{Pr}(\cdot)$ that the signal levels of all branches fall below a certain level, which using (2) can be expressed as

$$
F_{R}(r)=\prod_{k=1}^{L} \operatorname{Pr}\left(R_{k} \leq r\right)=\prod_{k=1}^{L}\left[1-\exp \left(-\frac{r^{\beta_{k}}}{\Omega_{k}}\right)\right] .
$$

We define the instantaneous input SNR per symbol in the $\ell$ th branch as $\gamma_{\ell}=R_{\ell}^{2} E_{s} / N_{0}$, where $E_{s}$ is the transmitted symbols energy and $N_{0}$ is the single-sided noise power spectral density (PSD) of the additive white Gaussian (AWGN), assumed identical and uncorrelated among the $L$ diversity branches. Hence, the average input SNR per symbol is $\bar{\gamma}_{\ell}=\mathcal{E}\left\langle R_{\ell}^{2}\right\rangle E_{s} / N_{0}$. Having assumed identical noise PSD to all diversity branches, the same branch will be also chosen, if the selection is based on the maximum instantaneous input SNR criterion. The instantaneous output SNR will be $\gamma_{\mathrm{sc}}=\max \left\{\gamma_{\ell}\right\}=R^{2} E_{s} / N_{0}$ with its PDF given by [10, eq. (4)]. That PDF can not be easily manipulated in its current form, and thus, after rearranging it, performing all the multiplications required, and setting $\beta_{\ell}=\beta, \forall \ell$, valid for practical applications, $[10,(4)]$ can be rewritten as

$$
\begin{aligned}
p_{\gamma_{\mathrm{sc}}}(\gamma)= & \frac{\beta}{2} \gamma^{\beta / 2-1} \\
& \times \sum_{k=1}^{L}(-1)^{k+1} \sum_{\lambda_{1}=1}^{L-k+1} \sum_{\lambda_{2}=\lambda_{1}+1}^{L-k+2} \cdots \sum_{\lambda_{k}=\lambda_{k-1}+1}^{L} \\
& \times \prod_{i=1}^{k} \exp \left[-\left(\frac{\gamma}{a \bar{\gamma}_{\lambda_{i}}}\right)^{\beta / 2}\right] \sum_{i=1}^{k} \frac{1}{\left(a \bar{\gamma}_{\lambda_{i}}\right)^{\beta / 2}}
\end{aligned}
$$




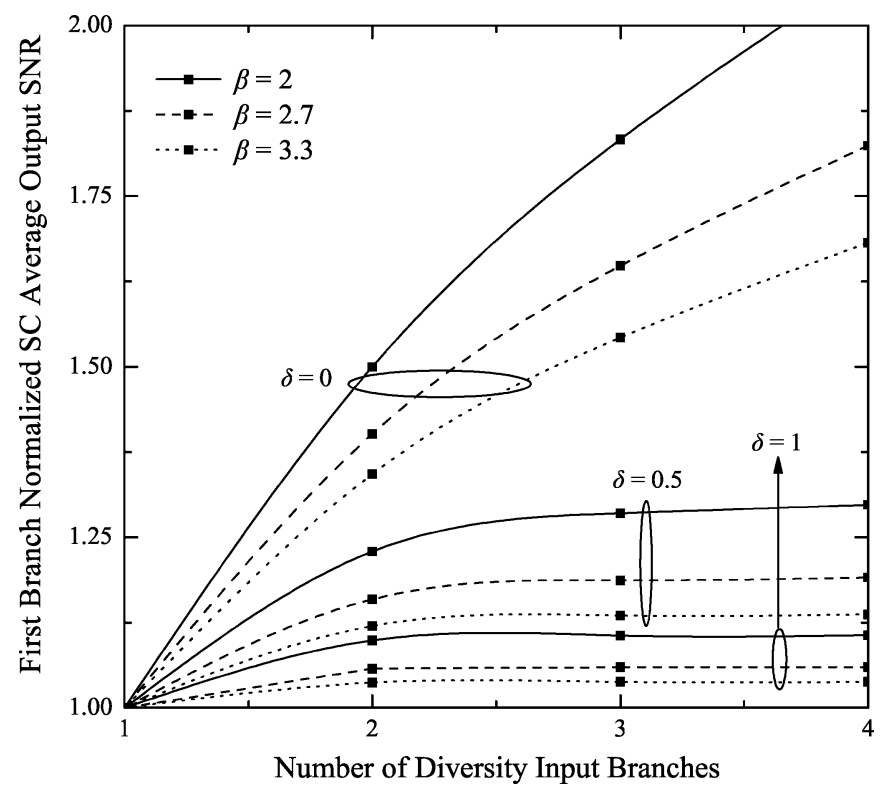

Fig. 1. First branch normalized average output SNR versus the number of diversity branches with an exponentially decaying PDP.

where $a=1 / \Gamma(1+2 / \beta)$. Note that (5) includes only sums of simple products of powers and exponential functions.

The $n$th order moment of the SC output SNR can be derived as $\mu_{n}=$ $\mathcal{E}\left\langle\gamma_{\mathrm{sc}}^{n}\right\rangle$. By substituting (5), interchanging the order of summation and integration, using [13, eq. (3.326/2)], and after some straightforward mathematical manipulations, $\mu_{n}$ can be obtained in closed form as

$$
\begin{aligned}
\mu_{n}= & \frac{\Gamma\left(1+\frac{2 n}{\beta}\right)}{\Gamma^{n}\left(1+\frac{2}{\beta}\right)} \sum_{k=1}^{L}(-1)^{k+1} \\
& \times \sum_{\lambda_{1}=1}^{L-k+1} \sum_{\lambda_{2}=\lambda_{1}+1}^{L-k+2} \ldots \sum_{\lambda_{k}=\lambda_{k-1}+1}^{L}\left(\sum_{i=1}^{k} \bar{\gamma}_{\lambda_{i}}^{-\beta / 2}\right)^{-2 n / \beta} .
\end{aligned}
$$

Note that for independent and identically distributed (i.i.d.) input branches, (6) reduces to [12, eq. (13)].

\section{A. Average Output SNR}

By setting $n=1$ in (6), the SC average output SNR with nonidentical input branches can be obtained in closed form as

$$
\bar{\gamma}_{\mathrm{sc}}=\mu_{1}
$$

while for i.i.d. input paths, $\bar{\gamma}_{\mathrm{sc}}$ reduces to [10, eq. (8)].

In Fig. 1, the first branch normalized average output SNR, $\bar{\gamma}_{\mathrm{sc}} / \bar{\gamma}_{1}$, is plotted as a function of the number of diversity input branches, $L$, for an exponentially decaying PDP $\bar{\gamma}_{\ell}=\bar{\gamma}_{1} \exp [-(\ell-1) \delta]$ and several values of $\beta$ and the power decaying factor $\delta \geq 0$. As expected, the diversity gain increases as $L$ increases, while for a fixed values of $\beta$ and $L$, the normalized average output SNR degrades rapidly as $\delta$ increases. Additionally, for a fixed value of $\delta$ the normalized output SNR increases as the severity of fading increases (i.e., as $\beta$ decreases).

\section{B. Amount of Fading}

The first two moments of $\gamma_{\mathrm{sc}}$ can be used in order to evaluate the AoF at the output of the combiner. The AoF is defined as the ratio of the variance to the square mean of $\gamma_{\mathrm{sc}}$ and can be expressed in closed form as

$$
A_{F}=\mu_{2} / \bar{\gamma}_{1}^{2}-1
$$

\section{AVERAGE LCR AND AFD}

The average LCR is defined as the average number of times per unit duration that the envelope of a fading channel crosses a given value in the negative direction and it can be evaluated as

$$
N(r)=\int_{0}^{\infty} \dot{r} p_{\dot{R}, R}(\dot{r}, r) d \dot{r}
$$

where $p_{\dot{R}, R}(\cdot, \cdot)$ is the joint PDF of $R$ and its time derivative $\dot{R}$. The AFD corresponds to the average length of time the envelope remains under a certain value once it crosses it in the negative direction and can be obtained as

$$
\tau(r)=\frac{F_{R}(r)}{N(r)} .
$$

Using (1), the time derivative of $R_{\ell}$ is

$$
\dot{R}_{\ell}=\frac{2}{\beta_{\ell}} R_{\ell}^{1-\beta_{\ell} / 2} \dot{X}_{\ell}
$$

where $\dot{X}_{\ell}$ is the time derivative of $X_{\ell}$. For isotropic scattering, $\dot{X}_{\ell}$ is Gaussian distributed with zero-mean and variance $\hat{\sigma}_{\ell}^{2}=\sigma_{\ell}^{2} 2 \pi^{2} f_{d}^{2}$, where $\sigma_{\ell}$ is the standard deviation of $X_{\ell}$ and $f_{d}$ is the maximum Doppler frequency shift. From (11), the PDF of $\dot{R}_{\ell}$ conditioned on $R_{\ell}$ is also a zero-mean Gaussian distribution, with standard deviation given by

$$
\hat{\sigma}_{R_{\ell}}=\frac{2}{\beta_{\ell}} R_{\ell}^{1-\beta_{\ell} / 2} \hat{\sigma}_{\ell} .
$$

Using (3), the time derivative of the envelope at the output of the SC receiver is $\dot{R}=\dot{R}_{i},\left(R_{i}=\max \left\{R_{\ell}\right\}\right)$ where using (11), it can be easily recognized that $\dot{R}$ conditioned on the $R_{\ell}$ is a zero-mean Gaussian distributed RV with variance given by $\hat{\sigma}_{R}^{2}=\hat{\sigma}_{R_{\ell}}^{2}$. If $\left(R_{i}=\right.$ $\max \left\{R_{\ell}\right\} \mid R_{i}=R$ ), the PDF of $\dot{R}$ is given by

$$
p_{\dot{R}}(\dot{r} \mid r)=\frac{1}{\sqrt{2 \pi} \hat{\sigma}_{R_{\ell}}} \exp \left(-\frac{\dot{r}^{2}}{2 \hat{\sigma}_{R_{\ell}}^{2}}\right) .
$$

Consequently, $\hat{\sigma}_{R}$ is a discrete RV with PDF

$$
\begin{aligned}
p_{\hat{\sigma}_{R}}\left(\hat{\sigma}_{r}\right) & =\sum_{i=1}^{L} P\left(\hat{\sigma}_{r}=\hat{\sigma}_{i}\right) \delta\left(\hat{\sigma}_{r}-\hat{\sigma}_{i}\right) \\
& =\sum_{i=1}^{L} \operatorname{Pr}\left(R_{i}=\max \left\{R_{\ell}\right\} \mid R_{i}=R\right) \delta\left(\hat{\sigma}_{r}-\hat{\sigma}_{i}\right)
\end{aligned}
$$

where $\delta(\cdot)$ is the Kronecker Delta function defined as $\delta(0)=1$ and zero otherwise. Using (9) and (13) and taking into account that $p_{\dot{R}, R}(\dot{r}, r)=p_{\dot{R}}(\dot{r} \mid r) p_{R}(r)$, the average LCR conditioned on $\hat{\sigma}_{R}$ is given by $N\left(r \mid \hat{\sigma}_{R}\right)=p_{R}(r) \hat{\sigma}_{R} / \sqrt{2 \pi}$. By averaging $N\left(r \mid \hat{\sigma}_{R}\right)$, i.e., $N(r)=\mathcal{E}\left\langle N\left(r \mid \hat{\sigma}_{R}\right)\right\rangle$, over the PDF of $\hat{\sigma}_{R}$ as determined in (14) and by using (12), yields

$$
\begin{aligned}
N(r)= & \sum_{i=1}^{L} p_{R_{i}}(r) \frac{\frac{2}{\beta_{\ell}} r^{1-\beta_{\ell} / 2} \hat{\sigma}_{\ell}}{\sqrt{2 \pi}} \\
& \times \operatorname{Pr}\left(R_{i}=\max \left\{R_{\ell}\right\} \mid R_{i}=R\right) .
\end{aligned}
$$


Taking into account the independence assumption between the input branches

$$
\operatorname{Pr}\left(R_{i}=\max \left\{R_{\ell}\right\} \mid R_{i}=R\right)=\prod_{\substack{k=1 \\ k \neq i}}^{L} F_{R_{k}}(r)
$$

the average LCR of the SC operating in Weibull fading can be obtained in closed form as

$$
\begin{aligned}
N(r)= & \sqrt{2 \pi} f_{d} \sum_{i=1}^{L} \frac{r^{\beta_{i} / 2}}{\Omega_{i}} \exp \left(-\frac{r^{\beta_{i}}}{\Omega_{i}}\right) \\
& \times \prod_{\substack{k=1 \\
k \neq i}}^{L}\left[1-\exp \left(-\frac{r^{\beta_{k}}}{\Omega_{k}}\right)\right] .
\end{aligned}
$$

By substituting (4) and (17) in (10), the AFD at the output of the $\mathrm{SC}$ can be also expressed in closed form. For i.i.d. input branches, after normalizing the signals levels to its root mean square (rms) value $\rho=r / r_{\text {rms }}$ with $r_{\text {rms }}=\sqrt{\mathcal{E}\left\langle R^{2}\right\rangle},(17)$ reduces to

$$
\begin{aligned}
N(\rho)= & L f_{d} \sqrt{2 \pi}\left(\frac{\rho}{\sqrt{a}}\right)^{\beta / 2} \exp \left[-\left(\frac{\rho}{\sqrt{a}}\right)^{\beta}\right] \\
& \times\left\{1-\exp \left[-\left(\frac{\rho}{\sqrt{a}}\right)^{\beta}\right]\right\}^{L-1} .
\end{aligned}
$$

Note that when $\beta=2$,(17) reduces to previously published expressions for the average LCR of the well-known Rayleigh model [6]. Furthermore, the maximum value of the average LCR can be derived solving $\partial N(r) /\left.\partial r\right|_{r=r_{\max }}=0$ and substituting $r_{\max }$ in (17). The case of $L=1$ has been addressed in [11]. Moreover, when $L>1$, well-known software mathematical packages such as Mathematica and Maple can be used for the numerical evaluation of $r_{\max }$. In particular, for i.i.d. input branches, using (18), the nonlinear equation

$$
\begin{aligned}
& \exp \left[\left(\frac{\rho_{\max }}{\sqrt{a}}\right)^{\beta / 2}\right]\left[2\left(\frac{\rho_{\max }}{\sqrt{a}}\right)^{\beta}-1\right] \\
& -2\left(\frac{\rho_{\max }}{\sqrt{a}}\right)^{\beta}-(L-1)\left(\frac{\rho_{\max }}{\sqrt{a}}\right)^{\beta / 2}+1=0
\end{aligned}
$$

is needed to be numerically solved with respect to $\rho_{\max }=r_{\max } / \sqrt{a}$.

Figs. 2 and 3 plot the average LCR normalized by $f_{d}, N(\rho) / f_{d}$, and AFD normalized by $1 / f_{d}, N(\rho) \times f_{d}$, respectively, for a dual-branch $\mathrm{SC}$ with i.i.d. input branches SNRs as a function of the normalized envelope level, $20 \log _{10}(\rho)$, for several values of $\beta$. As expected, when the fading severity increases the normalized average LCR increases, which means that the signal envelope fluctuates more frequently. Moreover, lower signal levels are crossed less frequently, whereas higher signal level are crossed more frequently.

\section{ERror RATE PERFormance}

The most straightforward approach to obtain the ASEP, $\bar{P}_{s e}$, is to average the conditional symbol error probability, $P_{s e}(\gamma)$, over the PDF of the combiner output SNR [1], i.e.,

$$
\bar{P}_{s e}=\int_{0}^{\infty} P_{s e}(\gamma) p_{\gamma_{s c}}(\gamma) d \gamma
$$

where $P_{\text {se }}(\gamma)$ can be written as follows.

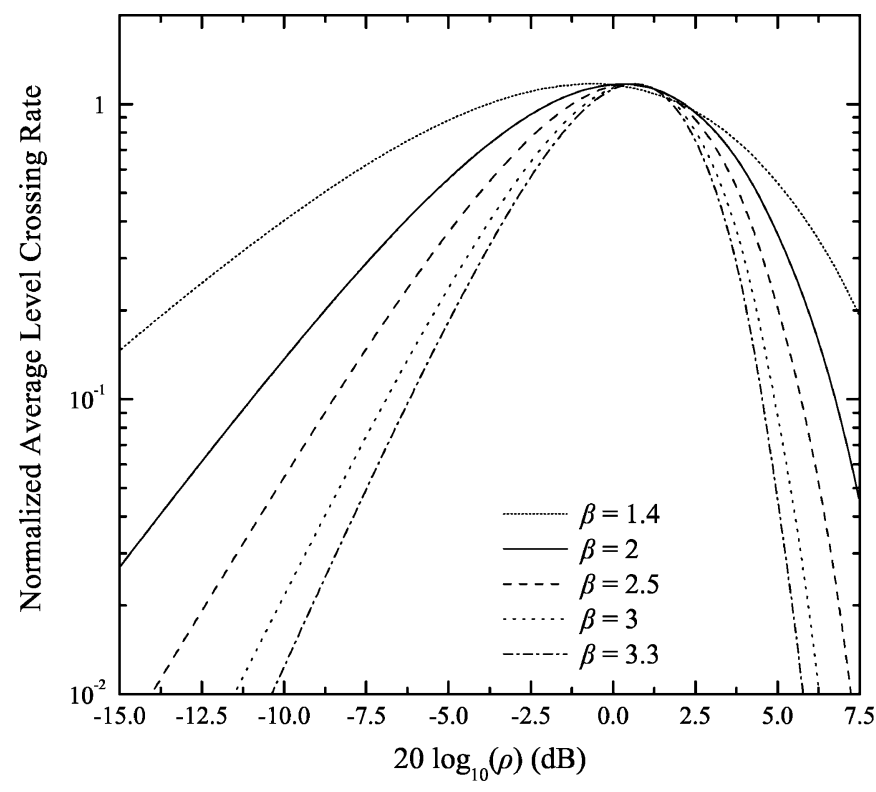

Fig. 2. Normalized average LCR versus normalized envelope level for a dualbranch SC receiver.

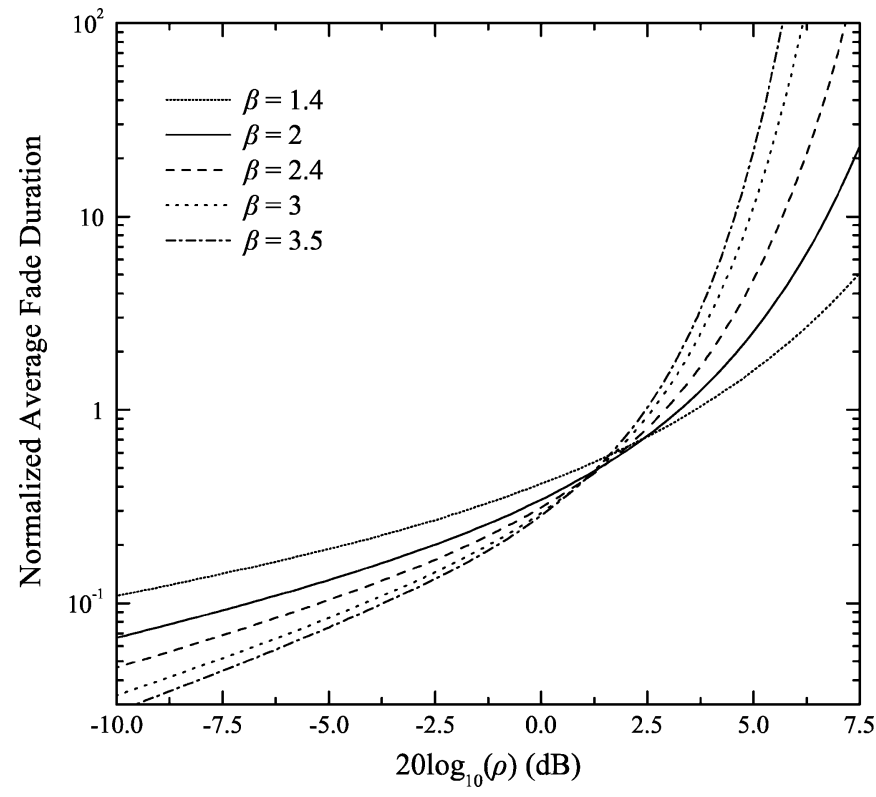

Fig. 3. Normalized AFD versus normalized envelope level for a dual-branch $\mathrm{SC}$ receiver.

1) For binary phase shift keying (BPSK), binary frequency shift keying (BFSK) and for high values of $\bar{\gamma}_{\ell}$ 's for Gaussian minimum shift keying ${ }^{1}$ (GMSK), $M$-ary-differentially encoded phase shift keying ( $M$-DEPSK), quadrature phase shift keying (QPSK), $M$ ary-phase shift keying ( $M$-PSK), $M$-ary-frequency shift keying ( $M$-FSK), square $M$-ary-quadrature amplitude modulation ( $M$ QAM), and $M$-ary-differential PSK ( $M$-DPSK) in the form of $P_{s e}(\gamma)=A \operatorname{erfc}(\sqrt{B \gamma})$, where $\operatorname{erfc}(\cdot)$ is the complementary error function [13, eq. (8.250/4)].

${ }^{1} \mathcal{B}$ is determined by the bandwidth of the premodulation Gaussian filter. 
TABLE I

Parameters $A$ AND $B$ For SeVeral Signaling Constellations

\begin{tabular}{c||c|c|c}
\hline \hline Modulation Scheme & $A$ & $B$ & $M$ \\
\hline \hline BPSK & $1 / 2$ & 1 & - \\
BFSK & $1 / 2$ & $1 / 2$ & - \\
\hline GMSK & 1 & $\mathcal{B}$ & - \\
$M$-DEPSK & 2 & $\sin ^{2}(\pi / M)$ & $\geq 2$ \\
QPSK & 1 & $1 / 2$ & - \\
$M$-PSK & 1 & $\sin ^{2}(\pi / M)$ & $>4$ \\
$M$-FSK & $(\mathrm{M}-1) / 2$ & $1 / 2$ & $>2$ \\
Square $M$-QAM & $2-2 / \sqrt{M}$ & $\frac{3}{2(M-1)}$ & $\geq 4$ \\
$M$-DPSK & 1 & $2 \sin ^{2}[\pi /(2 M)]$ & $\geq 2$ \\
\hline \hline DBPSK & $1 / 2$ & 1 & - \\
$M$-NFSK & $\sum_{k=1}^{M-1} \frac{(-1)^{k+1}}{k+1}$ & $\frac{k}{k+1}$ & $\geq 2$ \\
\hline \hline
\end{tabular}

2) For differential binary PSK (DBPSK) and $M$-ary-noncoherent frequency shift keying ( $M$-NFSK), in the form of $P_{s e}(\gamma)=$ $A \exp (-B \gamma)$.

The particular values of $A$ and $B$ depend on the considered modulation schemes which are summarized in Table I. Into the following, $\bar{P}_{s e}$ is obtained in closed-form expressions for each one of the above two cases.

A. ASEP for GMSK, M-DEPSK, M-PSK, M-FSK, Square $M-Q A M$, and $M$-DPSK

Using (5), it can be easily recognized that for coherent BPSK and BFSK and for higher values of average input SNR for GMSK, $M$-DEPSK, $M$-PSK, $M$-FSK, square $M$-QAM, and $M$-DPSK, the evaluation of integrals of the form $\Upsilon_{1}(\xi)=$ $\int_{0}^{\infty} x^{\beta / 2-1} \operatorname{erfc}(\sqrt{B x}) \exp \left(-\xi x^{\beta / 2}\right) d x$ is required, where $\xi$ is a positive real value. Since the above integral is not a tabulated one, a solution in terms of the Meijer's G-function [13, eq. (9.301)] has been given in [9], helping us to express the ASEP in a closed form as shown in (20) at the bottom of the page, where $\Delta(\cdot, \cdot)$ is defined as $\Delta(\mu, h)=h / \mu,(h+1) / \mu, \ldots,(h+\mu-1) / \mu$, with $\mu$ being positive integer and $h$ real constant and $G[\cdot]$ is the Meijer's G-function. The $\kappa$ and $\mu$ are positive integers such that

$$
\frac{\mu}{\kappa}=\frac{\beta}{2}
$$

holds. Depending upon the value of $\beta$, a set with minimum values of $\kappa$ and $\mu$ can be properly chosen in order (21) to be valid (e.g., for $\beta=2.6$ we have to choose $\kappa=10$ and $\mu=13$ ). For i.i.d. input paths, (20) reduces to

$$
\begin{aligned}
& \bar{P}_{s e}=A \frac{\beta L}{2(a \bar{\gamma})^{\beta / 2}} \frac{\sqrt{\kappa} \mu^{\beta / 2-1} B^{-\beta / 2}}{\sqrt{\pi}(\sqrt{2 \pi})^{\kappa+\mu-2}} \\
& \times \sum_{n=0}^{L-1}\left(\begin{array}{c}
L-1 \\
n
\end{array}\right)(-1)^{n} \\
& \times G_{2 \mu, \kappa+\mu}^{\kappa, 2 \mu}\left[\frac{(n+1)^{\kappa}}{\kappa^{\kappa}(a \bar{\gamma})^{\kappa \beta / 2}}\left(\frac{\mu}{B}\right)^{\mu} \mid \begin{array}{l}
\Delta\left(\mu, \frac{1-\beta}{2}\right), \Delta\left(\mu, 1-\frac{\beta}{2}\right) \\
\Delta(\kappa, 0), \Delta\left(\mu,-\frac{\beta}{2}\right)
\end{array}\right]
\end{aligned}
$$

\section{B. ASEP for DBPSK and $M-N F S K$}

For non-coherent modulation schemes such as DBPSK and $M$-NFSK, integrals with infinite limits of the form $\Upsilon_{2}(\xi)=$ $\int_{0}^{\infty} x^{\beta / 2-1} \exp (-B x) \exp \left(-\xi x^{\beta / 2}\right) d x$ should be evaluated. This type of integrals, has been analytically solved in terms of the Meijer's G-function [9] and the ASEP can be obtained in closed form as shown in (23) at the bottom of the page. Integers $\kappa$ and $\mu$ must be chosen so that (21) holds. For i.i.d. input branches, (23) can be reduced as

$$
\begin{aligned}
& \bar{P}_{s e}=\frac{\beta L A}{2(a \bar{\gamma})^{\beta / 2}} \sqrt{\frac{\kappa}{\mu}} \frac{\mu^{\beta / 2} B^{-\beta / 2}}{(\sqrt{2 \pi})^{\kappa+\mu-2}} \sum_{n=0}^{L-1}\left(\begin{array}{c}
L-1 \\
n
\end{array}\right)
\end{aligned}
$$

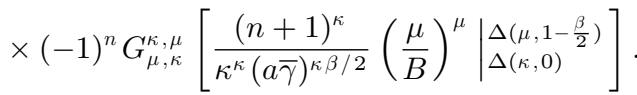

$$
\begin{aligned}
\bar{P}_{s e}= & A \frac{\beta \sqrt{\kappa} \mu^{\beta / 2-1} B^{-\beta / 2}}{2 \sqrt{\pi}(\sqrt{2 \pi})^{\kappa+\mu-2}} \sum_{n=1}^{L}(-1)^{n+1} \sum_{\lambda_{1}=1}^{L-n+1} \sum_{\lambda_{2}=\lambda_{1}+1}^{L-n+2} \cdots \sum_{\lambda_{n}=\lambda_{n-1}+1}^{L} \sum_{i=1}^{n}\left(a \bar{\gamma}_{\lambda_{i}}\right)^{-\beta / 2} \\
& \times G_{2 \mu, \kappa+\mu}^{\kappa, 2 \mu}\left[\left[\frac{1}{\kappa} \sum_{j=1}^{n}\left(a \bar{\gamma}_{\lambda_{j}}\right)^{-\beta / 2}\right]^{\kappa}\left(\frac{\mu}{B}\right)^{\mu} \mid \begin{array}{l}
\Delta\left(\mu, \frac{1-\beta}{2}\right), \Delta\left(\mu, 1-\frac{\beta}{2}\right) \\
\Delta(\kappa, 0), \Delta\left(\mu,-\frac{\beta}{2}\right)
\end{array}\right]
\end{aligned}
$$

$$
\begin{aligned}
\bar{P}_{s e}= & \sqrt{\frac{\kappa}{\mu}} \frac{A \beta \mu^{\beta / 2} B^{-\beta / 2}}{2(\sqrt{2 \pi})^{\kappa+\mu-2}} \sum_{n=1}^{L}(-1)^{n+1} \sum_{\lambda_{1}=1}^{L-n+1} \sum_{\lambda_{2}=\lambda_{1}+1}^{L-n+2} \cdots \sum_{\lambda_{n}=\lambda_{n-1}+1}^{L} \sum_{i=1}^{n}\left(a \bar{\gamma}_{\lambda_{i}}\right)^{-\beta / 2} \\
& \times G_{\mu, \kappa}^{\kappa, \mu}\left[\left[\frac{1}{\kappa} \sum_{j=1}^{n}\left(a \bar{\gamma}_{\lambda_{j}}\right)^{-\beta / 2}\right]^{\kappa}\left(\frac{\mu}{B}\right)^{\mu} \mid \begin{array}{l}
\Delta\left(\mu, 1-\frac{\beta}{2}\right) \\
\Delta(\kappa, 0)
\end{array}\right]
\end{aligned}
$$




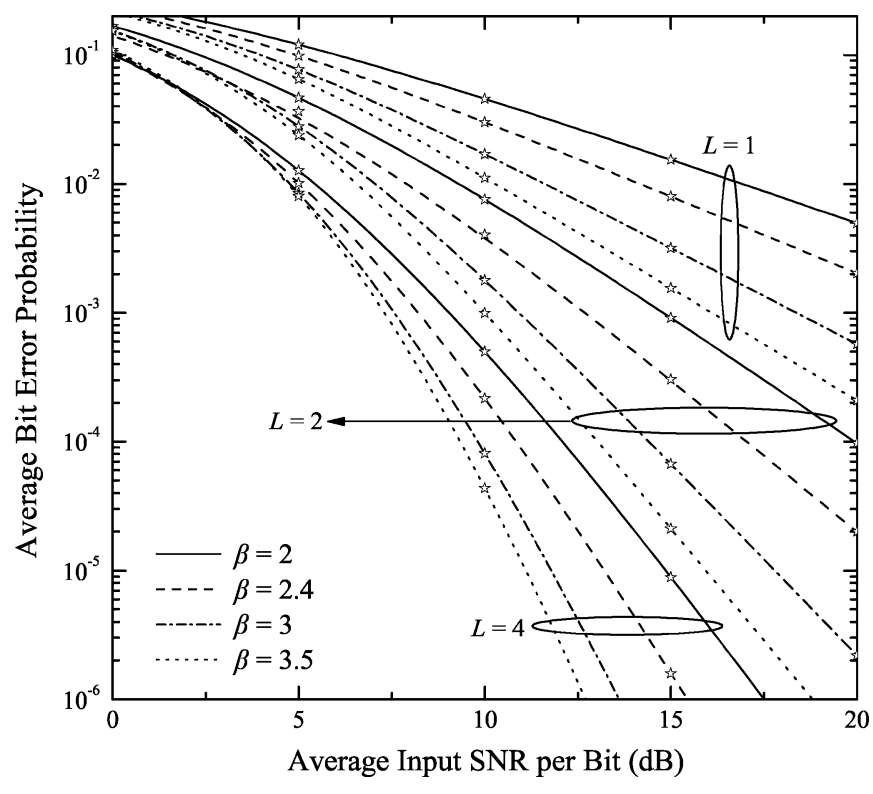

Fig. 4. Error performance of DBPSK employing SC in Weibull fading.

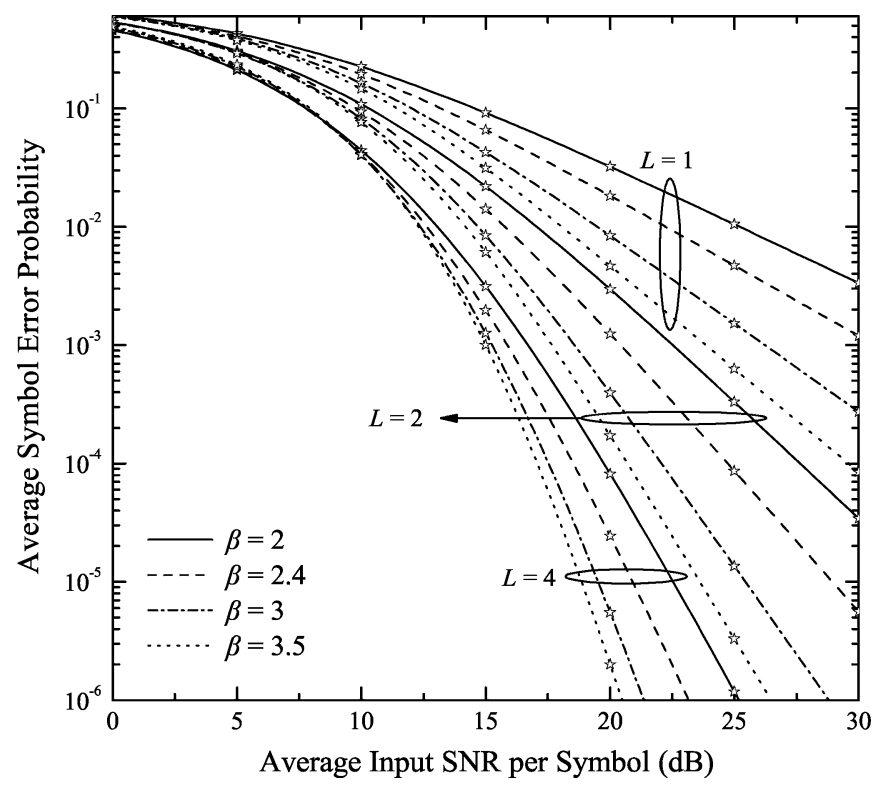

Fig. 5. Error performance of 8-PSK employing SC in Weibull fading.

\section{Numerical and Computer Simulation Results}

As typical examples, the ASEPs of DBPSK and 8-PSK are plotted in Figs. 4 and 5, respectively, as a function of the average input SNR per symbol for i.i.d. $\left(\bar{\gamma}_{\ell}=\bar{\gamma}\right)$ input branches and several values of $\beta$ and $L$. These results show that the error performance improves with the increase of $\beta$ and $L$. In order to verify these analytical results, computer simulations have been also performed and included in both figures (star symbols) for comparison purposes. Clearly, an excellent match between them is observed.

\section{ChanNel CAPACITY ANALYSIS}

The channel capacity, in the Shannon's sense, is an important performance measure since it provides the maximum achievable transmission rate under which the errors are recoverable. The average channel capacity can be expressed as [15]

$$
\bar{C}=\mathrm{BW} \int_{0}^{\infty} \log _{2}(1+\gamma) p_{\gamma_{\mathrm{sc}}}(\gamma) d \gamma
$$

where BW is the transmitted signal bandwidth. By substituting (5) in (25), an integral of the form $\int_{0}^{\infty} x^{\beta / 2-1} \ln (1+x) \exp \left(-\xi x^{\beta / 2}\right) d x$ is required to be evaluated. This type of integrals has been analytically solved in [11], and hence, the average channel capacity can be obtained in closed form as shown in (26) at the bottom of the page, where the values of integers $\kappa$ and $\mu$ must be chosen so that (21) holds. For i.i.d. input branches, (26) can be simplified to

$$
\begin{aligned}
& \bar{C}=\frac{L \beta \mathrm{BW}}{2(a \bar{\gamma})^{\beta / 2} \ln (2)} \frac{\sqrt{\kappa} / \mu}{(\sqrt{2 \pi})^{\kappa+2 \mu-3}} \sum_{n=0}^{L-1}\left(\begin{array}{c}
L-1 \\
n
\end{array}\right)(-1)^{n}
\end{aligned}
$$

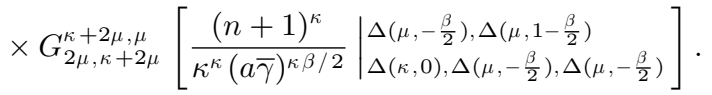

Note that by setting $L=1$, the above equation can be reduced to an already known expression [11, eq. (17)].

\section{CONCLUSION}

In this paper, important performance metrics of SC receivers, operating over independent, but not necessarily identically distributed, Weibull fading channels was addressed. Capitalizing on an extracted PDF formula of the SC output SNR, novel closed-form expressions for the average output SNR, AoF, ASEP for a broad class of digital modulations, and the Shannon average capacity were obtained. Furthermore, second order statistical criteria such as the average LCR and AFD were obtained for arbitrary parameters of the fading severity. Computer simulation results verified the accuracy and the correctness of the proposed analysis.

\section{REFERENCES}

[1] M. K. Simon and M.-S. Alouini, Digital Communication Over Fading Channels, 2nd ed., New York: Wiley, 2004.

[2] O. C. Ugweje, "Selection diversity for wireless communications in Nakagami-fading with arbitrary parameters," IEEE Trans. Veh. Technol., vol. 50, no. 6, pp. 1437-1449, Nov. 2001.

[3] K. Dietze, C. B. Dietrich, and W. L. Stutzman, "Analysis of a two-branch maximal and selection diversity system with unequal SNRs and correlated

$$
\begin{aligned}
\bar{C}= & \frac{\mathrm{BW} \beta \sqrt{\kappa} / \mu}{2 \ln (2)(\sqrt{2 \pi})^{\kappa+2 \mu-3}} \sum_{n=1}^{L}(-1)^{n+1} \sum_{\lambda_{1}=1}^{L-n+1} \sum_{\lambda_{2}=\lambda_{1}+1}^{L-n+2} \cdots \sum_{\lambda_{n}=\lambda_{n-1}+1}^{L} \sum_{i=1}^{n}\left(a \bar{\gamma}_{\lambda_{i}}\right)^{-\beta / 2} \\
& \times G_{2 \mu, \kappa+2 \mu}^{\kappa+2 \mu, \mu}\left[\left[\frac{1}{\kappa} \sum_{j=1}^{n}\left(a \bar{\gamma}_{\lambda_{j}}\right)^{-\beta / 2}\right]^{\kappa}\left(\frac{\mu}{B}\right)^{\mu} \mid \begin{array}{l}
\Delta\left(\mu,-\frac{\beta}{2}\right), \Delta\left(\mu, 1-\frac{\beta}{2}\right) \\
\Delta(\kappa, 0), \Delta\left(\mu,-\frac{\beta}{2}\right), \Delta\left(\mu,-\frac{\beta}{2}\right)
\end{array}\right]
\end{aligned}
$$


inputs for a Rayleigh fading channel," IEEE Trans. Wireless Commun., vol. 4, no. 1, pp. 274-281, Apr. 2002.

[4] Q. T. Zhang and H. G. Lu, "A general analytical approach to multi-branch selection combining over various spatially correlated fading channels," IEEE Trans. Commun., vol. 50, no. 7, pp. 1066-1073, Jul. 2002.

[5] G. K. Karagiannidis, D. A. Zogas, and S. A. Kotsopoulos, "Performance analysis of triple selection diversity over exponentially correlated Nakagami-m fading channels," IEEE Trans. Commun., vol. 51, no. 8, pp. 1245-1248, Aug. 2003.

[6] C.-D. Iskander and P. T. Mathiopoulos, "Analytical level-crossing rates and average fade durations for diversity techniques in Nakagami fading channels," IEEE Trans. Commun., vol. 50, no. 8, pp. 1301-1309, Aug. 2002.

[7] M. D. Yacoub, C. R. Cunha, M. da Silva, and J. E. V. Bautista, "Secondorder statistics for diversity-combining techniques in Nakagami-fading channels," IEEE Trans. Veh. Technol., vol. 50, no. 6, pp. 1464-1470, Nov. 2001.

[8] M.-S. Alouini and M. K. Simon, "Performance of generalized selection combining over Weibull fading channels," in Proc. IEEE Vehicular Technology Conference, vol. 3, Atlantic City, New Jersey, USA, Oct. 2001, pp. 1735-1739.
[9] N. C. Sagias, G. K. Karagiannidis, D. A. Zogas, P. T. Mathiopoulos, and G. S. Tombras, "Performance analysis of dual selection diversity in correlated Weibull fading channels," IEEE Trans. Commun., vol. 52, no. 7, pp. 1063-1067, Jul. 2004.

[10] N. C. Sagias, P. T. Mathiopoulos, and G. S. Tombras, "Selection diversity receivers in Weibull fading: Outage probability and average signal-tonoise ratio," Electron. Lett., vol. 39, no. 25, pp. 1859-1860, Dec. 2003.

[11] N. C. Sagias, D. A. Zogas, G. K. Karagiannidis, and G. S. Tombras, "Channel capacity and second order statistics in Weibull fading," IEEE Commun. Lett., vol. 8, no. 6, pp. 377-379, Jun. 2004.

[12] J. Cheng, C. Tellambura, and N. C. Beaulieu, "Performance of digital linear modulations on Weibull fading channels," IEEE Trans. Commun., vol. 52, no. 8, pp. 1265-1268, Aug. 2004.

[13] I. S. Gradshteyn and I. M. Ryzhik, Table of Integrals, Series, and Products, 6th ed., New York: Academic, 2000.

[14] M. D. Yacoub, "The $\alpha-\mu$ distribution: A general fading distribution," in Proc. IEEE Personal, Indoor, and Mobile Radio Communications, Lisbon, Portugal, Sep. 2002.

[15] F. Lazarakis, G. S. Tombras, and K. Dangakis, "Average channel capacity in a mobile radio enviroment with Rician statistics," IEICE Trans. Commun., vol. E77-B, no. 7, pp. 971-977, Jul. 1994. 